Research Article

\title{
Sharp Lower Bounds of the Sum-Connectivity Index of Unicyclic Graphs
}

\author{
Maryam Atapour \\ Department of Mathematics and Computer Science, Basic Science Faculty, University of Bonab, P.O. Box 55513-95133, \\ Bonab, Iran \\ Correspondence should be addressed to Maryam Atapour; m.atapour@ubonab.ac.ir
}

Received 1 August 2021; Revised 17 August 2021; Accepted 23 August 2021; Published 2 September 2021

Academic Editor: Ali Ahmad

Copyright (C) 2021 Maryam Atapour. This is an open access article distributed under the Creative Commons Attribution License, which permits unrestricted use, distribution, and reproduction in any medium, provided the original work is properly cited.

The sum-connectivity index of a graph $G$ is defined as the sum of weights $1 / \sqrt{d_{u}+d_{v}}$ over all edges $u v$ of $G$, where $d_{u}$ and $d_{v}$ are the degrees of the vertices $u$ and $v$ in graph $G$, respectively. In this paper, we give a sharp lower bound on the sum-connectivity index unicyclic graphs of order $n \geq 7$ and diameter $D(G) \geq 5$.

\section{Introduction and Preliminaries}

Let $G$ be a simple graph with a vertex set $V=V(G)$ and edge set $E(G)$. The integers $n=n(G)=|V(G)|$ and $m=m(G)=$ $|E(G)|$ are the order and the size of the graph $G$, respectively. The open neighborhood of vertex $v$ is $N_{G}(v)=N(v)=$ $\{u \in V(G) \mid u v \in E(G)\}$, and the degree of $v$ is $d_{G}(v)=d_{v}=$ $|N(v)|$. A pendant vertex is a vertex of degree one. The distance between two vertices is the number of edges in the shortest path connecting them, and the diameter $D(G)$ of $G$ is the distance between any two furthest vertices in $G$. A diametral path is the shortest path in $G$ connecting two vertices whose distance is $D(G)$. A unicyclic graph is a connected graph containing exactly one cycle. A subgraph $G^{\prime}$ of a graph $G$ is a graph whose set of vertices is a subset of $V(G)$, and set of edges is a subset of $E(G)$.

A topological index is a numeric number associated with a molecular graph that correlates certain physicochemical properties of chemical compounds. The topological indices are useful in the prediction of physicochemical properties and the bioactivity of the chemical compounds [1-3]. Also, topological indices invariants are used for Quantitative Structure-Activity Relationship (QSAR) and Quantitative Structure-Property Relationship (QSPR) studies. It was demonstrated that the sum-connectivity index is well correlated with a variety of physicochemical properties of alkanes, such as boiling point and enthalpy of formation. The sum-connectivity index is certainly the most widely applied in chemistry and pharmacology, in particular for designing quantitative structure-property and structure-activity relations. The sum-connectivity index is proposed to quantitatively characterize the degree of molecular branching.

Topological indices have been used and have been shown to give a high degree of predictability of pharmaceutical properties. The sum-connectivity index of a graph $G$ was proposed in [4] defined as follows:

$$
\operatorname{SCI}(G)=\sum_{u v \in E(G)} \frac{1}{\sqrt{d_{u}+d_{v}}} .
$$

The applications of the sum-connectivity index have been investigated in $[5,6]$. Some basic mathematical properties of the sum-connectivity index have been established in [4-8].

In [4], it was shown that for a graph $G$ with $n \geq 5$ vertices and without isolated vertices, $\operatorname{SCI}(G) \geq n-1 / \sqrt{n}$ with equality if and only if $G$ is the star. For $n=4$, this is not true since, for the union of two copies of the path on two vertices, its sum-connectivity index is $\sqrt{2}$, less than $3 / 2$. In [7], minimum sum-connectivity indices of trees and unicyclic graphs of a given matching number are characterized; in [8], sum-connectivity index of molecular trees are characterized; 
and in [4], some of the lower and upper bounds for the sumconnectivity index of trees are obtained (see recent bounds [9-13]). We all know that the sum-connectivity index is one of the most important and practical indices and therefore has been considered by many researchers. In this paper, we will address one of the unresolved issues for the sumconnectivity index. In fact, we investigate the relationship between the sum-connectivity index and diameter of the graph, which is one of the important parameters in graph theory and we get new results. In other words, in this paper, we solve the problem of the relationship between the diameter of a graph and the sum-connectivity index for the unicyclic graph.

\section{Main Results}

We begin with the following lemma that we will need for obtaining our main results.
We denote $t(G)$ as the number of pendant vertices in a graph $G$.

Lemma 1. Let $G$ be any unicyclic graph and $\mathbb{U}$ be a diametral path of $G$. If $G$ contains a pendant vertex $v$ not in $\mathbb{U}$, then there is a unicyclic graph $G^{\prime} \subset G$ not containing $v$, such that $D(G)=D\left(G^{\prime}\right), t\left(G^{\prime}\right)=t(G)-1$, and $S C I\left(G^{\prime}\right)<S C I(G)$.

Proof. Let $\mathbb{U}$ be a diametral path of $G$ and $v \in V(G)$ be a pendant vertex not in $\mathbb{U}$. We denote by $u$ the closest vertex to $v$ which is not of degree 2. Let $G^{\prime}$ be a subgraph of $G$ obtained by the removal of the path connecting $u$ and $v$ from $G$. Let $u^{\prime}$ be the neighbor of $u$ on the $u-v$ path (if the path has only one edge, then $u^{\prime}=v$ ). Clearly, $G^{\prime}$ is a unicyclic graph, $D\left(G^{\prime}\right)=D(G)$, and $t\left(G^{\prime}\right)=t(G)-1$. Furthermore,

$$
\operatorname{SCI}(G)-\operatorname{SCI}\left(G^{\prime}\right) \geq \frac{1}{\sqrt{\mathrm{d}(u)+1}}+\sum_{w \in N(u) \backslash\left\{u^{\prime}\right\}}\left(\frac{1}{\sqrt{\mathrm{d}(u)+\mathrm{d}(w)}}-\frac{1}{\sqrt{\mathrm{d}(u)+\mathrm{d}(w)-1}}\right)
$$

We know that the sum-connectivity index of the path $u v$ is at least $1 / \sqrt{\mathrm{d}(u)+1}$. Note that

$$
\frac{1}{\sqrt{\mathrm{d}(u)+\mathrm{d}(w)}}-\frac{1}{\sqrt{\mathrm{d}(u)+\mathrm{d}(w)-1}} \geq \frac{1}{\sqrt{\mathrm{d}(u)+1}}-\frac{1}{\sqrt{\mathrm{d}(u)}}
$$

for every $w \in N(u)\left\{u^{\prime}\right\}$; hence, we have

$$
\operatorname{SCI}(G)-\operatorname{SCI}\left(G^{\prime}\right) \geq \frac{1}{\sqrt{\mathrm{d}(u)+1}}+(\mathrm{d}(u)-1)\left(\frac{1}{\sqrt{\mathrm{d}(u)+1}}-\frac{1}{\sqrt{\mathrm{d}(u)}}\right) \text {. }
$$

Therefore, we get $\operatorname{SCI}(G)>\operatorname{SCI}\left(G^{\prime}\right)$.

By Lemma 1, it follows that for any unicyclic graph $G$, if $\mathbb{U}$ is a diametral path of $G$, then there is a unicyclic graph $G^{\prime} \subset G$ containing only pendant vertices of $\mathbb{U}$, where $D\left(G^{\prime}\right)=D(G)$ and $\operatorname{SCI}\left(G^{\prime}\right)<\operatorname{SCI}(G)$.

Here, we obtain a sharp bound on the sum-connectivity index of any unicyclic graph of diameter at least 5 .

Theorem 2. Let $G$ be any unicyclic graph of diameter $D(G) \geq 5$. Then,

$$
\operatorname{SCI}(G) \geq \frac{D(G)}{2}+\frac{5}{\sqrt{5}}+\frac{1}{\sqrt{3}}-2
$$

Proof. We will complete the proof by considering the following four cases.

Case 1 . If $G$ does not contain any pendant vertex, then $G$ is the cycle either with $2 D(G)$ or $2 D(G)+1$ vertices, which implies that

$$
\begin{aligned}
\operatorname{SCI}(G) \geq 2 D(G)\left(\frac{1}{\sqrt{4}}\right)= & D(G)>\frac{D(G)}{2} \\
& +\frac{5}{\sqrt{5}}+\frac{1}{\sqrt{3}}-2 .
\end{aligned}
$$

Case 2. If $G$ contains one pendant vertex, then $G$ consists of the cycle $\mathbb{C}_{r}$ of length $r \geq 3$ and the path $P$ having $s \geq 1$ edges, where $\mathbb{C}_{r} \cap P$ consists of one vertex which has degree 3 in $G$. This degree will be included in the computation of $\operatorname{SCI}\left(\mathbb{C}_{r}\right)$ and $\operatorname{SCI}(P)$. We have $\operatorname{SCI}(G)=\operatorname{SCI}\left(\mathbb{C}_{r}\right)+\operatorname{SCI}(P)$, where

$$
\begin{aligned}
\operatorname{SCI}\left(\mathbb{C}_{r}\right) & =\sum_{u v \in E\left(\mathbb{C}_{r}\right)} \frac{1}{\sqrt{\mathrm{d}(u)+\mathrm{d}(v)}}=(r-2) \frac{1}{\sqrt{4}}+2\left(\frac{1}{\sqrt{5}}\right)=\frac{r}{2}+\frac{2 \sqrt{5}}{5}-1, \\
\operatorname{SCI}(P) & =\sum_{u v \in E(P)} \frac{1}{\sqrt{\mathrm{d}(u)+\mathrm{d}(v)}}=(s-2) \frac{1}{\sqrt{4}}+\frac{1}{\sqrt{3}}+\frac{1}{\sqrt{5}}=\frac{s}{2}+\frac{\sqrt{3}}{3}+\frac{\sqrt{5}}{5}-1 .
\end{aligned}
$$


If $s \geq 2$ and $\operatorname{SCI}(P)=1 / 2$ if $s=1$. So, $\operatorname{SCI}(P) \geq s / 2$ for every $s \geq 1$, and the equality holds if $s=1$.

If $r \geq 4$, then $D(G) \leq(r / 2)+s$ and

$$
\begin{aligned}
\operatorname{SCI}(G) & \geq \frac{r}{2}+\frac{2 \sqrt{5}}{5}+\frac{s}{2}-1 \\
& \geq \frac{r}{2}+\frac{2 \sqrt{5}}{5}+\frac{D(G)}{2}-\frac{r}{4}-1 \\
& =\frac{D(G)}{2}+\frac{r}{4}+\frac{2 \sqrt{5}}{5}-1 \\
& \geq \frac{D(G)}{2}+\frac{2 \sqrt{5}}{5} \\
& >\frac{D(G)}{2}+\frac{5}{\sqrt{5}}+\frac{1}{\sqrt{3}}-2 .
\end{aligned}
$$

If $r=3$, then $D(G)=s+1$ and

$$
\begin{aligned}
\operatorname{SCI}(G) & \geq \frac{3}{2}+\frac{2 \sqrt{5}}{5}+\frac{s}{2}-1, \\
& \geq \frac{3}{2}+\frac{2 \sqrt{5}}{5}+\frac{D(G)}{2}-\frac{1}{2}-1 \\
& =\frac{D(G)}{2}+\frac{2 \sqrt{5}}{5} \\
& >\frac{D(G)}{2}+\frac{5}{\sqrt{5}}+\frac{1}{\sqrt{3}}-2 .
\end{aligned}
$$

Case 3. If $G$ contains 2 pendant vertices, then $G$ consists of the cycle $\mathbb{C}_{r}$ of length $r \geq 3$ and two paths $X_{1}$ and $X_{2}$ having $s_{1} \geq 1$ and $s_{2} \geq 1$ edges, respectively. We can assume that $\mathbb{C}_{r} \cap X_{1}$ consists of one vertex and $X_{2}$ is attached either to an internal vertex of $X_{1}$ or to a vertex of $\mathbb{C}_{r}$.

Case 3.1. If $X_{1} \cap X_{2}=\varnothing$, then we have $\operatorname{SCI}(G)=\operatorname{SCI}\left(\mathbb{C}_{r}\right)+\operatorname{SCI}\left(X_{1}\right)+\operatorname{SCI}\left(X_{2}\right)$. For $i=1,2$,

$$
\begin{aligned}
\operatorname{SCI}\left(X_{i}\right) & =\sum_{u v \in E\left(X_{i}\right)} \frac{1}{\sqrt{\mathrm{d}(u)+\mathrm{d}(v)}} \\
& =\left(s_{i}-2\right) \frac{1}{\sqrt{4}}+\frac{1}{\sqrt{3}}+\frac{1}{\sqrt{5}}=\frac{s_{i}}{2}+\frac{\sqrt{3}}{3}+\frac{\sqrt{5}}{5}-1 .
\end{aligned}
$$

When $s_{i} \geq 2$ and $\operatorname{SCI}\left(X_{i}\right)=1 / 2$ if $s_{i}=1$. If $X_{1}$ and $X_{2}$ are attached to nonadjacent vertices of $\mathbb{C}_{r}$, then

$$
\begin{aligned}
\operatorname{SCI}\left(\mathbb{C}_{r}\right) & =\sum_{u v \in E\left(\mathbb{C}_{r}\right)} \frac{1}{\sqrt{\mathrm{d}(u)+\mathrm{d}(v)}} \\
& =(r-4) \frac{1}{\sqrt{4}}+4\left(\frac{1}{\sqrt{5}}\right)=\frac{r}{2}+\frac{4 \sqrt{5}}{5}-2 .
\end{aligned}
$$

If $X_{1}$ and $X_{2}$ are attached to adjacent vertices of $\mathbb{C}_{r}$, then

$$
\begin{aligned}
\operatorname{SCI}\left(\mathbb{C}_{r}\right) & =\sum_{u v \in E\left(\mathbb{C}_{r}\right)} \frac{1}{\sqrt{\mathrm{d}(u)+\mathrm{d}(v)}}, \\
& =(r-3) \frac{1}{\sqrt{4}}+2\left(\frac{1}{\sqrt{5}}\right)+\frac{1}{\sqrt{6}} \\
& =\frac{r}{2}+\frac{2 \sqrt{5}}{5}+\frac{\sqrt{6}}{6}-\frac{3}{2} \\
& >\frac{r}{2}+\frac{4 \sqrt{5}}{5}-2 .
\end{aligned}
$$

If $s_{1}=s_{2}=1$, then $D(G) \leq(r / 2)+2$ and

$$
\begin{aligned}
\operatorname{SCI}(G) & \geq \frac{r}{2}+\frac{4 \sqrt{5}}{5}-2+\frac{1}{2}+\frac{1}{2} \\
& \geq D(G)+\frac{4 \sqrt{5}}{5}-3 \\
& >\frac{D(G)}{2}+\frac{5}{\sqrt{5}}+\frac{1}{\sqrt{3}}-2 .
\end{aligned}
$$

So, we can assume that $s_{1}$ or $s_{2}$ is at least 2 . We have $\operatorname{SCI}\left(X_{1}\right)+\operatorname{SCI}\left(X_{2}\right) \geq\left(s_{1} / 2\right)+\left(s_{2} / 2\right)+(\sqrt{3} / 3)+(\sqrt{5}$ 15) -1 (the equality holds if $s_{1}$ or $s_{2}$ is 1 ).

If $r \geq 4$, then $D(G) \leq(r / 2)+s_{1}+s_{2}$ and

$$
\begin{aligned}
\operatorname{SCI}(G) & \geq \frac{r}{2}+\frac{4 \sqrt{5}}{5}-2+\frac{s_{1}}{2}+\frac{s_{2}}{2}+\frac{\sqrt{3}}{3}+\frac{\sqrt{5}}{5}-1, \\
& \geq \frac{D(G)}{2}+\frac{r}{4}+\sqrt{5}+\frac{\sqrt{3}}{3}-3 \\
& \geq \frac{D(G)}{2}+\sqrt{5}+\frac{\sqrt{3}}{3}-2 .
\end{aligned}
$$

If $r=3$, then $D(G) \leq s_{1}+s_{2}+1$ and

$$
\begin{aligned}
\operatorname{SCI}(G) & \geq \frac{3}{2}+\frac{4 \sqrt{5}}{5}-2+\frac{s_{1}}{2}+\frac{s_{2}}{2}+\frac{\sqrt{3}}{3}+\frac{\sqrt{5}}{5}-1, \\
& \geq \frac{D(G)}{2}+\frac{4 \sqrt{5}}{5}+\frac{\sqrt{3}}{3}+\frac{\sqrt{5}}{5}-2 \\
& =\frac{D(G)}{2}+\sqrt{5}+\frac{\sqrt{3}}{3}-2 .
\end{aligned}
$$

Case 3.2. $X_{1} \cap X_{2}$ is nonempty, and there is a diametral path containing both pendant vertices of $G$.

Let $\mathbb{U}$ be the diametral path containing both pendant vertices of $G$. Then, $\mathbb{U} \subseteq X_{1} \cup X_{2}$. One of the internal vertices, say $x$, of $\mathbb{U}$ is of degree 3 or 4 in graph $G$. If $x$ is adjacent to a pendant vertex of $\mathbb{U}$, then 


$$
\begin{aligned}
\operatorname{SCI}(\mathbb{U}) & =\sum_{u v \in E(U)} \frac{1}{\sqrt{\mathrm{d}(u)+\mathrm{d}(v)}} \\
& =(D(G)-3) \frac{1}{\sqrt{4}}+\frac{1}{\sqrt{3}}+\frac{1}{\sqrt{5}}+\frac{1}{\sqrt{6}} \\
& =\frac{D(G)}{2}+\frac{\sqrt{3}}{3}+\frac{\sqrt{5}}{5}+\frac{\sqrt{6}}{6}-\frac{3}{2} .
\end{aligned}
$$

If $x$ is not adjacent to a pendant vertex of $\mathbb{U}$, then

$$
\begin{aligned}
\operatorname{SCI}(\mathbb{U}) & \geq \frac{D(G)-4}{2}+\frac{2 \sqrt{3}}{3}+\frac{2 \sqrt{6}}{6} \\
& =\frac{D(G)}{2}+\frac{2 \sqrt{3}}{3}+\frac{2 \sqrt{6}}{6}-2 .
\end{aligned}
$$

Note that $G$ contains also the cycle $\mathbb{C}_{r}$, where one of the vertices is of degree 3 or 4 in $G$. We have

$$
\begin{aligned}
\operatorname{SCI}\left(\mathbb{C}_{r}\right) & =\sum_{u v \in E\left(\mathbb{C}_{r}\right)} \frac{1}{\sqrt{\mathrm{d}(u)+\mathrm{d}(v)}} \\
& =(r-2) \frac{1}{\sqrt{4}}+2\left(\frac{1}{\sqrt{6}}\right) \geq \frac{3}{2}+\frac{2 \sqrt{6}}{6}-1 \\
& =\frac{1}{2}+\frac{2 \sqrt{6}}{6}
\end{aligned}
$$

which implies that

$$
\begin{aligned}
\operatorname{SCI}(G) & \geq \operatorname{SCI}(\mathbb{U})+\operatorname{SCI}\left(\mathbb{C}_{r}\right) \\
& \geq \frac{D(G)}{2}+\frac{\sqrt{3}}{3}+\frac{\sqrt{5}}{5}+\frac{\sqrt{6}}{6}-\frac{3}{2}+\frac{1}{2}+\frac{2 \sqrt{6}}{6}, \\
& =\frac{D(G)}{2}+\frac{\sqrt{3}}{3}+\frac{\sqrt{5}}{5}+\frac{\sqrt{6}}{2}-1 \\
& >\frac{D(G)}{2}+\frac{5}{\sqrt{5}}+\frac{1}{\sqrt{3}}-2 .
\end{aligned}
$$

Case 3.3. $X_{1} \cap X_{2}$ is nonempty and there is a diametral path containing only one pendant vertex of $G$. Then, we denote this diametral path with $\mathbb{U}$ containing only one pendant vertex of $G$. Since the other pendant vertex is not in $\mathbb{U}$, by Lemma 1 , there is a unicyclic graph $G^{\prime}$ having one pendant vertex, such that $D\left(G^{\prime}\right)=D(G)$ and $\operatorname{SCI}(G)>\operatorname{SCI}\left(G^{\prime}\right)$, and we know that $\operatorname{SCI}\left(G^{\prime}\right)>$ $(D(G) / 2)+(5 / \sqrt{5})+(1 / \sqrt{3})-2$.

Case 4 . If $G$ contains at least 3 pendant vertices.

Let $\mathbb{U}$ be a diametral path of $G$. Clearly, this path contains at most 2 pendant vertices of $G$. Since $G$ contains $m \geq 3$ pendant vertices, we have at least $m-2$ pendant vertices not in $\mathbb{U}$. By Lemma 1 , there is a unicyclic graph $G^{\prime} \subseteq G$ having only the pendant vertices of $\mathbb{U}$ (at most 2 vertices), such that $D\left(G^{\prime}\right)=D(G), t\left(G^{\prime}\right)=t(G)-1$ and $\operatorname{SCI}(G)>\operatorname{SCI}\left(G^{\prime}\right)$.
From the previous cases, it follows that $\operatorname{SCI}\left(G^{\prime}\right)>$ $(D(G) / 2)+(5 / \sqrt{5})+(1 / \sqrt{3})-2$.

It is easy to show that the bound $\operatorname{SCI}(G) \geq(D(G) / 2)+$ $(5 / \sqrt{5})+(1 / \sqrt{3})-2$ is best possible because of the graph $H$, where $V(H)=\left\{u, v_{0}, v_{1}, v_{2}, \ldots, v_{D(H)}\right\}$ and $E(H)=$ $\left\{v_{0} v_{1}, v_{1} v_{2}, \ldots, v_{D(H)-1} v_{D(H)}, u v_{1}, u v_{3}\right\}$ has the sum-connectivity index

$$
\begin{aligned}
\operatorname{SCI}(H) & =(D(H)-4) \frac{1}{\sqrt{4}}+5\left(\frac{1}{\sqrt{5}}\right)+\frac{1}{\sqrt{3}}, \\
& =\frac{D(H)}{2}+\frac{5}{\sqrt{5}}+\frac{1}{\sqrt{3}}-2 .
\end{aligned}
$$

The proof is completed.

Now, we obtain lower bounds on the sum-connectivity index for unicyclic graphs of small diameter.

Theorem 3. Let $G$ be an unicyclic graph of diameter $D(G)$. Then,

(i) If $D(G)=2$, then $S C I(G) \geq 1+2 \sqrt{5} / 5$

(ii) If $D(G)=3$, then $S C I(G) \geq 2 \sqrt{5} / 5+\sqrt{6} / 6+1$

(iii) If $D(G)=4$, then $S C I(G) \geq 1+4 \sqrt{5} / 5$

Proof. We can see that the proof of Theorem 2 holds for $D(G)=3$ and $D(G)=4$ except for Case 3.1, where $s_{1}=s_{2}=1$.

Let $D(G)=4$. We have SCI $(G) \geq D(G)+(1+4 \sqrt{5} / 5)-$ 3 (as presented in the proof of Theorem 2), which is $(1+4 \sqrt{5} / 5)$. From the other cases, we obtain SCI $(G) \geq((D$ $(G) / 2)+(5 / \sqrt{5})+(1 / \sqrt{3})-2)=(\sqrt{5}+(1 / \sqrt{3}))>(1+4$ $\sqrt{5} / 5)$, which implies that $\operatorname{SCI}(G) \geq(1+4 \sqrt{5} / 5)$.

Let $D(G)=3$. We have $\operatorname{SCI}(G) \geq D(G)+4 \sqrt{5} / 5-3$ (obtained in the proof of Theorem 2); Case 3.1 (if $s_{1}=s_{2}=1$ ) is not sufficient now, so we give a better bound in this case. Since $s_{1}=s_{2}=1$ and $D(G)=3$, then $P_{1}$ and $P_{2}$ must be attached to adjacent vertices of $C_{r}$, which means that $\operatorname{SCI}\left(C_{r}\right)=r / 2+2 \sqrt{5} / 5+\sqrt{6} / 6-3 / 2$ in the proof of Theorem 2 . Since $r \geq 3$, we obtain

$$
\begin{aligned}
\operatorname{SCI}(G) & =\operatorname{SCI}\left(C_{r}\right)+\operatorname{SCI}\left(X_{1}\right)+\operatorname{SCI}\left(X_{2}\right) \\
& =\frac{r}{2}+\frac{2 \sqrt{5}}{5}+\frac{\sqrt{6}}{6}-\frac{3}{2}+\frac{1}{2}+\frac{1}{2} \geq \frac{2 \sqrt{5}}{5}+\frac{\sqrt{6}}{6}+1 .
\end{aligned}
$$

Let $D(G)=2$. Except for $\mathbb{C}_{5}$ and $\mathbb{C}_{4}$, the only unicyclic graphs $H$ of diameter 2 are formed by the cycle $\mathbb{C}_{3}$, where $s \geq 1$ pendant vertices are adjacent to one of the vertices of $C_{3}$. Let $V\left(\mathbb{C}_{3}\right)=\left\{v_{1}, v_{2}, v_{3}\right\}$. We can assume that the pendant vertices $u_{1}, u_{2}, \ldots, u_{p}$ are adjacent to $v_{1}$. Then, $\mathbb{U}=v_{2} v_{1} u_{1}$ is a diametral path of $H$, and from Lemma 1, it follows that there is a unicyclic graph $H^{\prime} \subseteq H$, which contains only one pendant vertex $u_{1}$ (the pendant vertex (the pendant vertex in $\mathbb{U}))$, where $\operatorname{SCI}(H) \geq \operatorname{SCI}\left(H^{\prime}\right)$. Since 


$$
\operatorname{SCI}\left(H^{\prime}\right)=2\left(\frac{1}{\sqrt{5}}\right)+2\left(\frac{1}{\sqrt{4}}\right)=1+\frac{2 \sqrt{5}}{5}
$$

$\operatorname{SCI}\left(\mathbb{C}_{4}\right)=2$ and $\operatorname{SCI}\left(\mathbb{C}_{5}\right)=5 / 2$, we obtain the bound $\operatorname{SCI}(G) \geq 1+2 \sqrt{5} / 5$.

Corollary 4. Let $G$ be any unicyclic graph of order at least 7 and diameter $D(G) \geq 2$. Then,

$$
\operatorname{SCI}(G) \geq \frac{D(G)}{2}+\frac{5}{\sqrt{5}}+\frac{1}{\sqrt{3}}-2 .
$$

Proof. By Theorem 2, for $D(G) \geq 5$ and any $n$, we have $\operatorname{SCI}(G) \geq((D(G) / 2)+(5 / \sqrt{5})+(1 / \sqrt{3})-2)$. By Theorem 3 , for $D(G)=2$ and any $n$, we have $\operatorname{SCI}(G) \geq 1+2 \sqrt{5} / 5$, which is greater than $((D(G) / 2)+(5 / \sqrt{5})+(1 / \sqrt{3})-2)$. It remains to prove Corollary 4 for $n \geq 7$ and $3 \leq D(G) \leq 4$. The proof of Theorem 2 holds also for $D(G)=3$ and $D(G)=4$ except for Case 3.1 where $s_{1}=s_{2}=1$. We show that if $n \geq 7$, then $\operatorname{SCI}(G) \geq((D(G) / 2)+(5 / \sqrt{5})+(1 / \sqrt{3})-2)$ also in that case. If $n \geq 7$ and $p_{1}=p_{2}=1$, then $G$ contains the cycle $C_{k}$ for $r \geq 5$ and SCI $\left(\mathbb{C}_{r}\right) \geq r / 2+4 \sqrt{5} / 5-2$ (given in Case 3.1 in the proof of Theorem 2). Since SCI $\left(X_{1}\right)=\operatorname{SCI}\left(X_{2}\right)=1 / 2$, we obtain

$$
\operatorname{SCI}(G)=\operatorname{SCI}\left(C_{r}\right)+\operatorname{SCI}\left(X_{1}\right)+\operatorname{SCI}\left(X_{2}\right) \geq \frac{3}{2}+\frac{4 \sqrt{5}}{5},
$$

which is greater than $\operatorname{SCI}(G) \geq((D(G) / 2)+(5 / \sqrt{5})+(1 /$ $\sqrt{3})-2$ ) for $D(G)=3$ and $D(G)=4$.

Corollary 5. Let $G$ be any unicyclic graph of order at least 7 and diameter $D(G) \geq 2$. Then,

$$
\begin{array}{r}
\frac{\operatorname{SCI}(G)}{D(G)} \geq \frac{(\sqrt{3} / 3)+\sqrt{5}}{n-2}-\frac{1}{2}, \\
\operatorname{SCI}(G)-D(G) \geq \frac{\sqrt{3}}{3}+\sqrt{5}-\frac{n}{2}-1 .
\end{array}
$$

Proof. By Theorem 2, we have $\operatorname{SCI}(G) \geq(D(G) / 2)+(5 / \sqrt{5})+1 / \sqrt{3}-2$ and since $D(G) \leq n-2$ for any graph $G$ except for the path, hence, by the definition of sum-connectivity index, we have

$$
\begin{aligned}
\frac{\operatorname{SCI}(G)}{D(G)} & \geq \frac{1}{2}+\frac{5}{\sqrt{5} D(G)}+\frac{1}{\sqrt{3} D(G)}-\frac{2}{D(G)} \\
& \geq \frac{1}{2}+\frac{5}{\sqrt{5}(n-2)}+\frac{1}{\sqrt{3}(n-2)}-\frac{2}{D(G)} \\
& \geq \frac{1}{2}+\frac{5}{\sqrt{5}(n-2)}+\frac{1}{\sqrt{3}(n-2)}-1 \\
& =\frac{(\sqrt{3} / 3)+\sqrt{5}}{n-2}-\frac{1}{2} .
\end{aligned}
$$

Similarly, we obtain

$$
\operatorname{SCI}(G)-D(G) \geq \frac{\sqrt{3}}{3}+\sqrt{5}-\frac{D(G)}{2}-2 \geq \frac{\sqrt{3}}{3}+\sqrt{5}-\frac{n}{2}-1 \text {. }
$$

\section{Open Problem and Conclusion}

In this paper, we investigate the relationship between the sum-connectivity index and the diameter of a graph and obtained a new lower bound for the sum-connectivity index of unicyclic graphs. However, there are still open and challenging problems for researchers, for example, the problem on the relationship between the sum-connectivity index and the diameter of bicyclic and tricyclic graphs. Moreover, the relationship between other topological indices such as F-index and GA-index with the diameter of unicyclic, bicyclic, and tricyclic graphs is still open.

\section{Data Availability}

The data used to support the findings of the study are included within the article.

\section{Conflicts of Interest}

The author declares that there are no conflicts of interest.

\section{References}

[1] Z. Shao, M. Siddiqui, and M. Muhammad, "Computing zagreb indices and zagreb polynomials for symmetrical nanotubes," Symmetry, vol. 10, no. 7, p. 244, 2018.

[2] Z. Shao, P. Wu, X. Zhang, D. Dimitrov, and J.-B. Liu, "On the maximum $\mathrm{ABC}$ index of graphs with prescribed size and without pendent vertices," IEEE Access, vol. 6, pp. 2760427616, 2018.

[3] Z. Shao, P. Wu, Y. Gao, I. Gutman, and X. Zhang, "On the maximum $\mathrm{ABC}$ index of graphs without pendent vertices," Applied Mathematics and Computation, vol. 315, pp. 298-312, 2017.

[4] B. Zhou and N. Trinajstić, "On a novel connectivity index," Journal of Mathematical Chemistry, vol. 46, no. 4, pp. 12521270, 2009.

[5] B. Lučić, S. Nikolič, N. Trinajstić, B. Zhou, and S. Ivaniš Turk, "Sum-Connectivity index," in Novel Molecular Structure Descriptors - Theory and Applications I, I. Gutman and B. Furtula, Eds., pp. 101-136, University of Kragujevac, Kragujevac, Serbia, 2010.

[6] B. Lučić, N. Trinajstić, and B. Zhou, "Comparison between the sum-connectivity index and product-connectivity index for benzenoid hydrocarbons," Chemical Physics Letters, vol. 475, pp. 146-148, 2009.

[7] Z. Du, B. Zhou, and N. Trinajstić, "Minimum sum-connectivity indices of trees and unicyclic graphs of a given matching number," Journal of Mathematical Chemistry, vol. 47, no. 2, pp. 842-855, 2010.

[8] R. Xing, B. Zhou, and N. Trinajstić, "Sum-connectivity index of molecular trees," Journal of Mathematical Chemistry, vol. 48, no. 3, pp. 583-591, 2010. 
[9] S. Akram, M. Javaid, and M. Jamal, "Bounds on F-index of tricyclic graphs with fixed pendant vertices," Open Mathematics, vol. 18, no. 1, pp. 150-161, 2020.

[10] A. Ali, L. Zhong, and I. Gutman, "Harmonic index and its generalizations: extremal results and bounds," MATCH Communications in Mathematical and in Computer Chemistry, vol. 81, no. 2, pp. 249-311, 2019.

[11] W. Carballosa, D. Pestana, J. M. Sigarreta, and E. Tourís, "Relations between the general sum connectivity index and the line graph," Journal of Mathematical Chemistry, vol. 58, no. 10, pp. 2273-2290, 2020.

[12] A. Jahanbani, "Two-tree graphs with minimum sum-connectivity index," Discrete Mathematics, Algorithms and Applications, vol. 13, Article ID 2150053, 2020.

[13] M. Rizwan, A. A. Bhatti, M. Javaid, and F. Jarad, "Some bounds on bond incident degree indices with some parameters," Mathematical Problems in Engineering, vol. 2021, Article ID 8417486, 10 pages, 2021. 